\title{
Active phenomena in the circumstellar environment of the Herbig Ae star HD 31648
}

\author{
N. G. Beskrovnaya ${ }^{1,2}$ and M. A. Pogodin ${ }^{1,2}$ \\ ${ }^{1}$ Central Astronomical Observatory of the Russian Academy of Science at Pulkovo, Pulkovo 65-1, \\ 196140 Saint-Petersburg, Russia \\ e-mail: pogodin@gao.spb.ru \\ ${ }^{2}$ Isaac Newton Institute of Chile, St.Petersburg Branch, Russia
}

Received 10 July 2002 / Accepted 18 September 2003

\begin{abstract}
We present the results of observations of the Herbig Ae star HD 31648 including high-resolution spectroscopy near the $\mathrm{H} \alpha$, He I $\lambda 5876 \AA$ and DNa I lines, multi-color photometry and polarimetry. These observations have revealed many features of the observational appearance of HD 31648 similar to those of the "classical" Herbig Ae stars. Among them are spectral manifestations of both the mass loss and the disk accretion onto the star and certain types of line profile variations. The totality of the observed properties of HD 31648 provides evidence in favor of complicated structure in the stellar wind zone, exhibiting latitudinal stratification and containing local inhomogeneities in the form of outflowing streams and density condensations. Structural variations of the stellar wind have been found to correlate with changes in the accretion process in the envelope. The circumstellar environment of HD 31648 is supposed to contain dust particles of different forms manifesting themselves, in particular, in the variable multi-component intrinsic polarization.
\end{abstract}

Key words. line: profiles - polarization - stars: circumstellar matter - stars: individual: HD 31648 - stars: mass loss stars: pre-main sequence

\section{Introduction}

\subsection{Observational programme}

This investigation was carried out within the framework of a complex programme of the study of structural and kinematical peculiarities in the gaseous envelopes around the pre-main sequence Herbig Ae/Be stars (HAEBEs) and related objects. The method involved is based on a search for profile variability of the lines originating in different circumstellar (CS) regions. Analysis of these variations opens the way to reconstructing the density and velocity distributions throughout the envelope and to investigate characteristics of the interaction between the star and its CS envelope. Additional independent parameters of CS media can be obtained from the multi-color photometry and polarimetry accompanying the spectroscopic observations.

The previous targets of our programme were as follows: HD 163296 (Pogodin 1994; Beskrovnaya et al. 1998), HD 200775 (Beskrovnaya et al. 1994; Pogodin et al. 2000), AB Aur (Beskrovnaya et al. 1995), HD 50138 (Pogodin 1997), HD 36112 (Beskrovnaya et al. 1999), and HD 100546 (Vieira et al. 1999).

Send offprint requests to: N. G. Beskrovnaya, e-mail: beskr@gao.spb.ru
Our study has shown that all the objects from our list possess CS envelopes with inhomogeneous latitudinal structure variable in time. They display signs of both stellar wind and matter infall onto the star. The stellar wind of these stars contains outflowing streams and density condensations. Local gaseous-dusty inhomogeneities are likely to rotate inside the envelope.

The goal of this paper is to perform a detailed analysis of the CS environment of the next object of our programme, HD 31648.

\subsection{Background information}

HD 31648 (MWC 480, A2/3ep+sh, $V=7^{\mathrm{m}} \cdot 6$ ) is a peculiar Ae star with a long history of study. A variable emission line spectrum of the object was first described by Merrill et al. (1942).

HD 31648 is situated in the Taurus-Auriga complex of dark clouds where a local group of Herbig $\mathrm{Ae} / \mathrm{Be}$ stars and candidates is arranged in a chain. Among them are the well-known objects AB Aur, RR Tau, HD 36112 as well as the poorly studied Ae stars HD 32509, HD 35187. The reddening of the object is rather small $\left(A_{\mathrm{v}}=0 . \mathrm{m} 25\right.$, van den Ancker et al. 1998). Herbig (1994) classified HD 31648 as: "the star whose vicinity is quite 
unobscured, but for which there are obscuration nebulosities 1-2 degrees away".

Allen \& Swings (1976) included HD 31648 in the list of peculiar $\mathrm{Ae} / \mathrm{Be}$ stars with infrared (IR) excess connected to radiation of CS dust. The IR excess of the object was investigated by Sitko (1981). He noted that the energy distribution of HD 31648 is similar to that of classical Ae Herbig stars (e.g., AB Aur), except for the $9.7 \mu \mathrm{m}$ silicate feature in the spectrum of HD 31648 being slightly weaker. But he also mentioned that the IR emission of the object may be variable. Later on Sitko et al. (1999) and Chen et al. (2000) analyzed the spectrum of HD 31648 in the interval $3-14 \mu \mathrm{m}$ obtained with higher resolution $(\Delta \lambda \sim 0.2 \mu \mathrm{m})$ and concluded that in both size and structure the $9.7 \mu \mathrm{m}$ silicate feature resembles those observed in the pre-main sequence (PMS) Herbig Ae/Be stars and in solar system comets.

The spectrum of HD 31648 in the photographic region was studied in detail by Jaschek et al. (1988), who described a complicated behavior of the Balmer lines. In contrast to $\mathrm{H} \beta$, displaying the PCyg type profile, the other lines of this series (up to at least H 9) demonstrate a complex structure with an asymmetric diffuse absorption profile, accompanied by additional emission and shell components. The authors mentioned dissimilarity with results of an earlier study by Burbidge \& Burbidge (1954), who reported a similar P Cyg structure for all Balmer line profiles from $\mathrm{H} \beta$ to $\mathrm{H} 10$, a presence of many metallic shell lines from Fe I, Fe II, Ti II, Ca II, and Sc II which were not revealed by Jaschek et al. (1988).

The spectrum of the object in the near IR $(\lambda 7570-$ $-8830 \AA$ A) was discussed in follow-up papers by Jaschek et al. (1991, 1993). The IR Ca II triplet and the O I lines (at $\lambda 7772$ and $8446 \AA$ ) have been observed in emission, while they are usually absent in the spectra of classical Ae stars. At the same time, the spectra of peculiar B[e] stars contain as a rule numerous Fe II lines which are not seen in HD 31648. The authors conclude that the near IR spectrum of HD 31648 resembles that of a Herbig Ae star.

Studies in other spectral regions confirm the similarity of HD 31648 and already recognized HAEBEs. In particular, the IUE UV low-resolution spectra of the object contain the resonance $\mathrm{Mg}$ II doublet with a distinctive P Cyg type profile, several emission lines of O I, O II, Si II, Si III, Si IV, and C IV as well as absorption Fe II lines, thus resembling the UV spectra of AB Aur, HD 163296, and HD 190073 (Sitko et al. 1981; Imhoff 1994; Grady et al. 1996).

In the enlarged catalogue of Thé et al. (1994) HD 31648 is also classified as a candidate Herbig Ae star. The position of HD 31648 in the HR diagram corresponds to a PMS object with the age of about $2-4 \times 10^{6}$ years (van den Ancker et al. 1998). Mannings \& Sargent (1997) and Mannings et al. (1997) have mapped the thermal dust millimeter-continuum and gaseous CO emission towards HD 31648 and found that a CS disk surrounding HD 31648 has an extent of $85 \mathrm{AU}$ (FWHM) and an inclination angle $i \approx 30^{\circ}$. Pérez \& Grady (1998) have estimated $i$ as $43^{\circ}$.

The basic goal of our work was to collect new highresolution spectroscopic data for HD 31648 and to compare the results with those for similar objects. The following envelope lines were selected for observations:

a) the $\mathrm{HeI} \lambda 5876 \AA$ line generated in the high-temperature region near the star; b) the DNaI doublet lines at $\lambda 5890$ and $5896 \AA$ originating in the outer parts of the envelope, and c) the $\mathrm{H} \alpha$ line, which is forming throughout the envelope. Measurements of the brightness and linear polarization parameters in the UBVRI passbands of the Johnson system accompanied the spectral observations during some seasons.

\section{Observations and data reduction}

\subsection{Spectroscopic observations}

The spectroscopic observations of HD 31648 were carried out at the Crimean Astrophysical Observatory during five seasons: (I) two nights in November, 1996; (II) three nights in March-April, 1997; (III) four nights in March-April, 1998; (IV) five nights in October, 1998; and (V) three nights in March, 1999.

The 2.6m Shajn telescope of the Crimean Astrophysical Observatory equipped with a coudé-spectrograph was used for observations. The CCD system - SDS-9000 "Photometric $G m b H$ " mounted in the first camera of the spectrograph yields a resolving power $R \sim 30000$ and the wavelength coverage $\Delta \lambda$ of about $65 \AA$ near the $\mathrm{H} \alpha$ line (the second order of grating).

60 spectra have been collected in three spectral regions encompassing the lines $\mathrm{H} \alpha, \mathrm{H} \beta, \mathrm{HI}$ and DNaI (centered at $\lambda 5885 \AA$ ). In addition, two spectra have been obtained near the Si II lines at $\lambda 6347$ and $6371 \AA$. Data reduction followed the standard procedures and was done with the SPE code developed at the Crimean Observatory by Sergeev.

The details of observations such as dates, spectral lines, UT corresponding to the middle of an exposure, and $S / N$ ratio are collected in Table 1 .

\subsection{UBVRI photometry and polarimetry}

The spectroscopy of HD 31648 was accompanied by photometric and polarimetric observations during three seasons (November, 1996; March-April, 1998, and October, 1998) at the Crimean Astrophysical Observatory. The five-channel photometer/polarimeter of the Helsinki University (Piirola 1975; Efimov et al. 1984) attached to the $1.25 \mathrm{~m}$ AZT-11 telescope was used. This instrument enables the brightness and linear polarization parameters of an object to be monitored simultaneously in five passbands close to the standard Johnson UBVRI system. The diaphragm of $10^{\prime \prime}-20^{\prime \prime}$ was used depending on seeing conditions. The exposure time was $10 \mathrm{~s}$, resulting in 3.5 min being necessary for one polarimetric measurement. The sky background was recorded every $15 \mathrm{~min}$ for $30 \mathrm{~s}$ and then interpolated and subtracted from every $10 \mathrm{~s}$ integration in each channel. A correction of polarimetric data for instrumental polarization was performed with the use of the special observations of unpolarized standards from the catalogue of Serkowski (1974). The normalized Stokes parameters $(q=Q / I$ and $u=U / I$ ) were computed for each individual measurement and then averaged (as the weighted mean) for each observing night. 
Table 1. Observational log of spectroscopic data, including the UT of mid-exposure and the signal-to-noise ratio $(S / N)$ at the continuum level for each spectrum.

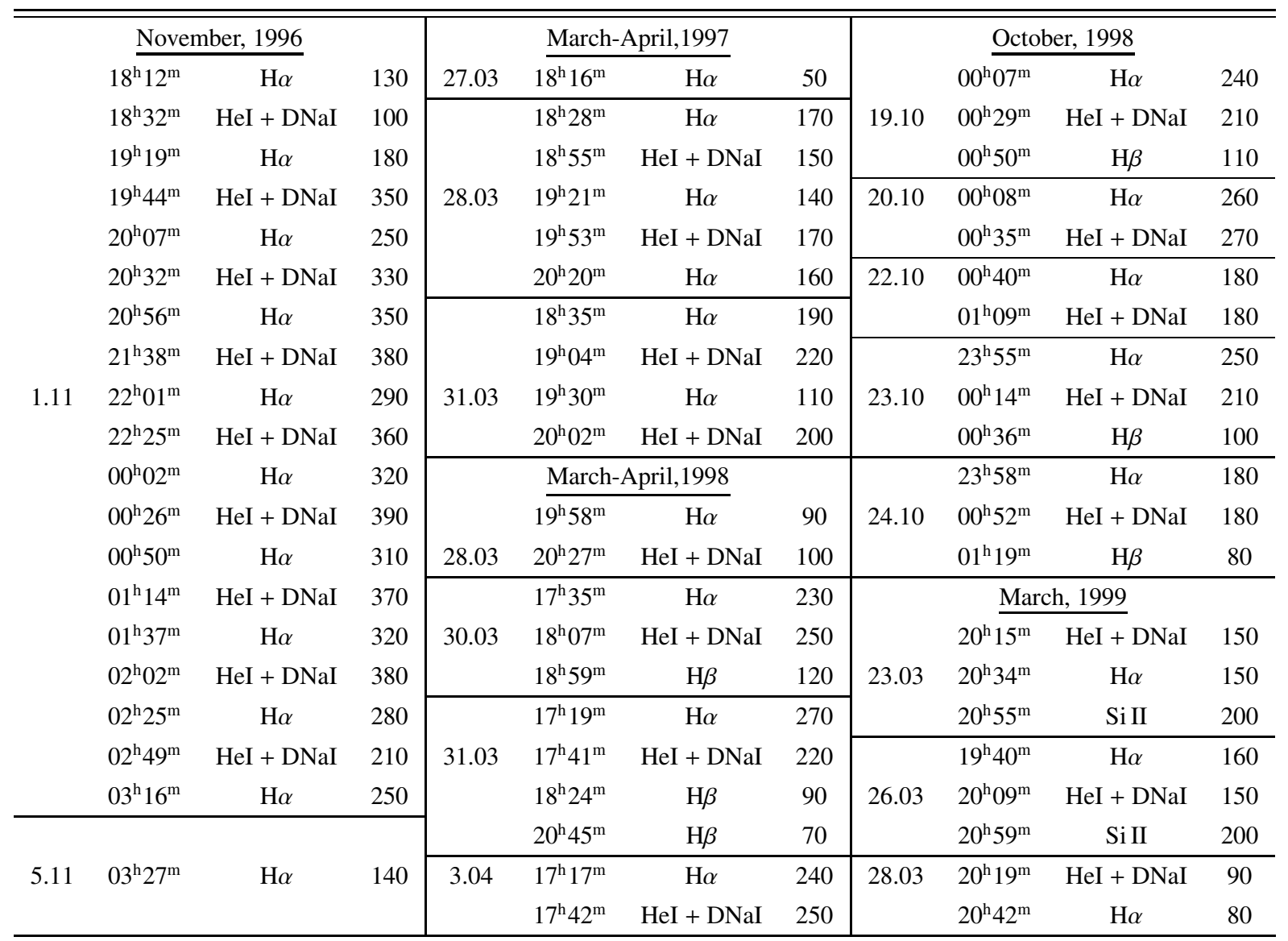

The log of polarimetric observations together with the polarization parameters obtained for each Julian Date (the percentage $P \%=\sqrt{q^{2}+u^{2}}$ and the position angle $\theta=$ $0.5 \arctan q / u$ ) are presented in Table 2. The results of our $U B V R I$ photometry are presented in Table 3 . Typical errors of these measurements are $0.01-0.02$.

\section{Results}

\subsection{Photometry}

As seen in Table 3, HD 31648 was photometrically stable during all seasons of our observations in 1996-1998. The amplitude of variations does not exceed $0 \mathrm{~m} 05$ in $B, R$ and $I$ and is somewhat higher in the $U$ passband. The correlation coefficients between variations in the $U, B, R, I$ and $V$ passbands are $0.65 \pm 0.14,0.86 \pm 0.06,0.75 \pm 0.10$, and $0.62 \pm 0.15$, respectively, suggesting the presence of a low-amplitude variability, which can be also seen in comparison with Hipparcos data (the average values $V=7.73, B-V=0.16$, relating to the early nineties). The mean magnitudes presented in Table 3 are in good agreement with the photometric data provided by Miroshnichenko, which were obtained in January 1995-December 1996: $V=7 \mathrm{~m} .65, U-B=0.15$, $B-V=0.15, V-R=0 . \mathrm{m} 20$, and $V-I=0.30$.
In contrast to magnitudes, the colors do not demonstrate variations exceeding the photometric errors and do not correlate with the $V$-magnitude.

\subsection{Spectral behavior}

On the majority of observing dates the $\mathrm{H} \alpha$ emission line in the spectrum of HD 31648 displays (with the only exception in October 1998) the P Cyg profile of type III with a secondary blue emission peak (Beals 1951). At the same time, profiles of other prominent CS lines, the He I line and the DNa I doublet have a rather complex structure. The He I line possesses a two-component profile including a blue emission peak changing its intensity over a wide range down to zero, and a strongly variable red part. At different moments this part of the profile appears alternately to be absorption or emission. The variations observed in both parts of the profile are not correlated one with each other. The DNa I lines demonstrate the most striking and complicated structure variable in time. Most frequently the DNa I lines display the P Cyg profile of type II (without a secondary emission, Beals 1951) with numerous local features overlapping the blue absorption component.

The behavior of the $\mathrm{H} \alpha, \mathrm{He} \mathrm{I}$ and DNaI lines in the spectrum of HD 31648 during each observing season is discussed in detail in the next sections. The profiles of these 
Table 2. Weighted mean linear polarization parameters of HD 31648. $N$ is the number of individual measurements in each data point, $\sigma_{\mathrm{p}}$ and $\sigma_{\theta}$ are the estimated errors of the weighted mean values of the polarization degree and of the position angle, respectively.

\begin{tabular}{|c|c|c|c|c|c|c|c|c|c|c|c|c|c|}
\hline $\begin{array}{c}\text { Date/ } \\
\text { JD2 } 400000+\end{array}$ & $N$ & Band & $\begin{array}{l}P \\
\%\end{array}$ & $\begin{array}{l}\sigma_{\mathrm{p}} \\
\%\end{array}$ & $\theta^{\circ}$ & $\sigma_{\theta}^{\circ}$ & $\begin{array}{c}\text { Date/ } \\
\text { JD } 2400000+\end{array}$ & $N$ & Band & $\begin{array}{l}P \\
\%\end{array}$ & $\begin{array}{l}\sigma_{\mathrm{p}} \\
\%\end{array}$ & $\theta^{\circ}$ & $\sigma_{\theta}^{\circ}$ \\
\hline 01.11 .96 & 40 & $\bar{U}$ & 0.094 & 0.014 & 14.3 & 5.1 & 07.11 .96 & 18 & $\bar{U}$ & 0.198 & 0.020 & 32.9 & 3.6 \\
\hline \multirow[t]{4}{*}{50389.39} & & $B$ & 0.205 & 0.009 & 33.3 & 1.7 & 50395.33 & & $B$ & 0.192 & 0.012 & 53.9 & 2.2 \\
\hline & & $V$ & 0.243 & 0.012 & 39.6 & 1.8 & & & V & 0.312 & 0.017 & 49.5 & 2.4 \\
\hline & & $R$ & 0.305 & 0.009 & 42.6 & 0.9 & & & $R$ & 0.310 & 0.012 & 51.4 & 1.2 \\
\hline & & $I$ & 0.317 & 0.012 & 46.4 & 1.2 & & & $I$ & 0.334 & 0.017 & 52.0 & 1.4 \\
\hline 01.11 .96 & 49 & $U$ & 0.134 & 0.013 & 25.3 & 3.8 & 07.11 .96 & 8 & $U$ & 0.108 & 0.025 & 48.3 & 8.1 \\
\hline \multirow[t]{4}{*}{50389.58} & & $B$ & 0.209 & 0.008 & 39.4 & 1.7 & 50395.62 & & $B$ & 0.220 & 0.015 & 51.9 & 2.5 \\
\hline & & V & 0.252 & 0.011 & 37.7 & 1.6 & & & $V$ & 0.280 & 0.021 & 49.3 & 2.4 \\
\hline & & $R$ & 0.324 & 0.008 & 41.6 & 1.0 & & & $R$ & 0.337 & 0.015 & 51.1 & 1.3 \\
\hline & & $I$ & 0.358 & 0.011 & 44.0 & 1.0 & & & $I$ & 0.381 & 0.021 & 57.0 & 1.5 \\
\hline 04.11 .96 & 24 & $U$ & 0.212 & 0.019 & 19.3 & 3.4 & 08.11 .96 & 25 & $U$ & 0.126 & 0.16 & 39.5 & 5.8 \\
\hline \multirow[t]{4}{*}{50392.33} & & $B$ & 0.233 & 0.012 & 41.7 & 1.5 & 50396.46 & & $B$ & 0.198 & 0.010 & 50.6 & 2.2 \\
\hline & & $V$ & 0.264 & 0.015 & 44.4 & 1.9 & & & V & 0.236 & 0.014 & 48.6 & 2.0 \\
\hline & & $R$ & 0.309 & 0.011 & 45.4 & 1.5 & & & $R$ & 0.321 & 0.011 & 53.4 & 1.4 \\
\hline & & $I$ & 0.339 & 0.015 & 48.0 & 1.4 & & & $I$ & 0.325 & 0.014 & 57.1 & 1.8 \\
\hline 04.11 .96 & 32 & $U$ & 0.073 & 0.014 & 22.4 & 6.3 & 09.11 .96 & 52 & $U$ & 0.092 & 0.018 & 36.0 & 8.9 \\
\hline \multirow[t]{4}{*}{50392.41} & & $B$ & 0.220 & 0.009 & 45.4 & 1.7 & 50397.37 & & $B$ & 0.181 & 0.011 & 59.2 & 3.2 \\
\hline & & V & 0.227 & 0.012 & 45.9 & 1.9 & & & V & 0.187 & 0.014 & 62.4 & 3.7 \\
\hline & & $R$ & 0.336 & 0.009 & 47.1 & 1.3 & & & $R$ & 0.282 & 0.011 & 59.8 & 2.4 \\
\hline & & $I$ & 0.381 & 0.013 & 49.8 & 1.4 & & & $I$ & 0.371 & 0.015 & 58.9 & 1.8 \\
\hline 04.11 .96 & 16 & $U$ & 0.175 & 0.021 & 26.6 & 4.5 & 28.03 .98 & 8 & $U$ & 0.238 & 0.044 & 14.4 & 8.1 \\
\hline \multirow[t]{4}{*}{50392.62} & & $B$ & 0.197 & 0.013 & 48.7 & 2.9 & 50901.31 & & $B$ & 0.254 & 0.025 & 37.1 & 4.2 \\
\hline & & $V$ & 0.247 & 0.018 & 47.0 & 2.2 & & & $V$ & 0.358 & 0.031 & 48.4 & 3.0 \\
\hline & & $R$ & 0.299 & 0.013 & 50.1 & 2.0 & & & $R$ & 0.336 & 0.031 & 50.8 & 2.2 \\
\hline & & $I$ & 0.376 & 0.018 & 48.7 & 1.8 & & & $I$ & 0.372 & 0.029 & 37.7 & 3.4 \\
\hline 05.11 .96 & 23 & $U$ & 0.195 & 0.016 & 35.9 & 3.4 & 30.03 .98 & 14 & $U$ & 0.337 & 0.030 & 34.7 & 7.7 \\
\hline \multirow[t]{4}{*}{50393.50} & & $B$ & 0.186 & 0.010 & 47.0 & 1.9 & 50903.27 & & $B$ & 0.288 & 0.017 & 35.1 & 3.8 \\
\hline & & V & 0.265 & 0.014 & 49.4 & 1.6 & & & V & 0.308 & 0.021 & 42.5 & 2.3 \\
\hline & & $R$ & 0.327 & 0.011 & 50.9 & 1.3 & & & $R$ & 0.297 & 0.016 & 43.3 & 2.3 \\
\hline & & $I$ & 0.360 & 0.015 & 52.5 & 1.2 & & & $I$ & 0.309 & 0.020 & 43.8 & 2.5 \\
\hline 05.11 .96 & 36 & $U$ & 0.111 & 0.013 & 37.2 & 4.5 & 31.03 .98 & 9 & $U$ & 0.119 & 0.048 & 42.3 & 14.2 \\
\hline \multirow[t]{4}{*}{50393.59} & & $B$ & 0.229 & 0.008 & 47.8 & 1.5 & 50904.26 & & $B$ & 0.308 & 0.025 & 43.1 & 3.3 \\
\hline & & V & 0.281 & 0.011 & 49.0 & 1.5 & & & $V$ & 0.245 & 0.030 & 44.2 & 6.1 \\
\hline & & $R$ & 0.343 & 0.009 & 53.6 & 1.0 & & & $R$ & 0.395 & 0.021 & 49.3 & 4.3 \\
\hline & & $I$ & 0.396 & 0.012 & 53.4 & 1.0 & & & $I$ & 0.531 & 0.028 & 51.4 & 3.2 \\
\hline 06.11 .96 & 12 & $U$ & 0.233 & 0.037 & 48.7 & 4.9 & 01.04 .98 & 8 & $U$ & 0.100 & 0.033 & 30.4 & 14.2 \\
\hline \multirow[t]{4}{*}{50394.32} & & $B$ & 0.244 & 0.022 & 49.1 & 3.6 & 50905.25 & & $B$ & 0.277 & 0.020 & 41.3 & 3.4 \\
\hline & & V & 0.188 & 0.029 & 35.1 & 5.0 & & & V & 0.278 & 0.026 & 43.1 & 2.3 \\
\hline & & $R$ & 0.334 & 0.022 & 55.5 & 2.2 & & & $R$ & 0.343 & 0.019 & 52.2 & 2.2 \\
\hline & & $I$ & 0.354 & 0.030 & 48.3 & 3.0 & & & $I$ & 0.381 & 0.026 & 51.1 & 2.2 \\
\hline
\end{tabular}

lines are presented in Figs. 1-3. As in previous papers, we have used the narrow interstellar (IS) (and, in part, CS) components for a definition of the radial velocity rest frame connected to the star. In general, the position of these features with respect to the solar system is slightly shifted from season to season: $+17.0 \mathrm{~km} \mathrm{~s}^{-1}$ (XI.96), $+18.1 \mathrm{~km} \mathrm{~s}^{-1}$ (III-IV.97), +14.0 km s${ }^{-1}$ (III-IV.98), $+14.2 \mathrm{~km} \mathrm{~s}^{-1}$ (X.98), and $+14.1 \mathrm{~km} \mathrm{~s}^{-1}$ (III.99) with typical errors $\pm \sigma=$ $0.2-0.3 \mathrm{~km} \mathrm{~s}^{-1}$. But these differences are too small to influence the accuracy of our radial velocity scale. 
Table 2. Weighted mean linear polarization parameters of HD 31648 (continued).

\begin{tabular}{ccccccc}
\hline \hline $\begin{array}{c}\text { Date/ } \\
\text { JD 2 400000+ }\end{array}$ & $N$ & Band & $\begin{array}{c}P \\
\%\end{array}$ & $\begin{array}{c}\sigma_{\mathrm{p}} \\
\%\end{array}$ & $\theta^{\circ}$ & $\sigma_{\theta}{ }^{\circ}$ \\
\hline 22.10 .98 & \multirow{2}{*}{16} & $U$ & 0.231 & 0.031 & 47.4 & 6.1 \\
51109.57 & & $B$ & 0.405 & 0.021 & 48.3 & 2.7 \\
& & $V$ & 0.381 & 0.027 & 54.6 & 1.8 \\
& & $R$ & 0.458 & 0.020 & 51.6 & 1.9 \\
& & $I$ & 0.440 & 0.027 & 49.4 & 3.2 \\
\hline 24.10 .98 & 18 & $U$ & 0.237 & 0.028 & 49.3 & 2.1 \\
51111.52 & & $B$ & 0.414 & 0.020 & 52.4 & 1.7 \\
& & $V$ & 0.382 & 0.025 & 52.7 & 2.0 \\
& & $R$ & 0.418 & 0.018 & 53.6 & 1.8 \\
& & $I$ & 0.416 & 0.025 & 56.3 & 2.2 \\
\hline 28.10 .98 & 12 & $U$ & 0.289 & 0.026 & 50.2 & 4.9 \\
51115.59 & & $B$ & 0.347 & 0.017 & 54.4 & 2.9 \\
& & $V$ & 0.282 & 0.023 & 54.6 & 2.8 \\
& & $R$ & 0.312 & 0.016 & 55.6 & 2.9 \\
& & $I$ & 0.420 & 0.023 & 51.2 & 3.2 \\
\hline
\end{tabular}

Table 3. UBVRI photometry of HD 31648. The Julian dates are given with the offset of 2400000 .

\begin{tabular}{cccccc}
\hline \hline $\mathrm{JD}$ & $V$ & $(U-B)$ & $(B-V)$ & $(V-R)$ & $(V-I)$ \\
\hline 50389.42 & 7.64 & 0.14 & 0.11 & 0.19 & 0.33 \\
50389.58 & 7.62 & 0.15 & 0.11 & 0.16 & 0.33 \\
50392.36 & 7.63 & 0.16 & 0.12 & 0.18 & 0.33 \\
50392.61 & 7.64 & 0.16 & 0.11 & 0.16 & 0.33 \\
50394.30 & 7.66 & 0.17 & 0.11 & 0.16 & 0.33 \\
50395.33 & 7.65 & 0.16 & 0.12 & 0.16 & 0.33 \\
50395.62 & 7.64 & 0.15 & 0.12 & 0.16 & 0.32 \\
50396.46 & 7.63 & 0.18 & 0.11 & 0.17 & 0.32 \\
50397.39 & 7.64 & 0.17 & 0.12 & 0.18 & 0.32 \\
50901.31 & 7.65 & 0.14 & 0.10 & 0.20 & 0.31 \\
50903.25 & 7.66 & 0.17 & 0.11 & 0.18 & 0.35 \\
50904.31 & 7.68 & 0.19 & 0.15 & 0.16 & 0.35 \\
50905.25 & 7.67 & 0.15 & 0.12 & 0.17 & 0.33 \\
51106.60 & 7.65 & 0.17 & 0.12 & 0.17 & 0.33 \\
51107.61 & 7.65 & 0.14 & 0.11 & 0.17 & 0.33 \\
51111.48 & 7.67 & 0.10 & 0.15 & 0.19 & 0.36 \\
51115.61 & 7.65 & 0.17 & 0.14 & 0.19 & 0.35 \\
\hline Mean & 7.65 & 0.15 & 0.12 & 0.17 & 0.33 \\
\hline
\end{tabular}

Two spectra of HD 31648 obtained near the photospheric Si II lines in March 1999 (see Table 1) were used by Kozlova et al. (2004) as additional data for the determination of the projected rotation velocity of the object $V \sin i=85 \pm 5 \mathrm{~km} \mathrm{~s}^{-1}$.

\subsubsection{November 1996}

Figure 1 illustrates the nightly averaged profiles of the $\mathrm{H} \alpha$, $\mathrm{He} \mathrm{I}$ and DNa I lines $(a, b)$. The $\mathrm{H} \alpha$ line has a typical P Cyg III profile with a small additional peak inside the blue-shifted absorption. Intensities and velocities of all the components including the central and secondary blue peaks and the blue absorption with its centrally located fine structures demonstrate small changes from November 1 to November 5. The nightly mean He I profile looks like a single blue-shifted emission peak. The DNa I line has the P Cyg II profile with three local absorptions situated near $-55,-100$, and near $-150 \mathrm{~km} \mathrm{~s}^{-1}$. The last changed its position from -140 to $-155 \mathrm{~km} \mathrm{~s}^{-1}$ over $8 \mathrm{~h}$. The positions of the blue and red edges of the profiles in this season were: $\pm 450 \mathrm{~km} \mathrm{~s}^{-1}$ (for $\mathrm{H} \alpha$ ), $\pm 400 \mathrm{~km} \mathrm{~s}^{-1}$ (for He I), and $\pm 200 \mathrm{~km} \mathrm{~s}^{-1}$ (for lines of the DNa I doublet).

Rapid variability of these lines during one observing night (on November, 1) is illustrated in Fig. 5. The He I line is seen to display an inverse P Cyg-type profile with a stable symmetric emission peak and a red symmetric absorption component monotonically increasing its depth during the night (left panel).

The variability of the $\mathrm{H} \alpha$ profile during this night is shown in the middle panel of Fig. 5, which presents the residuals constructed with respect to the nightly mean profile. A monotonous intensity rise of a local spectral feature centered at $-260 \mathrm{~km} \mathrm{~s}^{-1}$ without positional shift is clearly seen. In addition, a number of spectral details are observed in both red and central parts, moving bluewards with constant rate. Similar "standing waves" on the residual DNa I profiles are also seen in their blue parts (right panel).

\subsubsection{March-April 1997}

Variations of the same line profiles during this season are shown in Figs. 1c,d. The behavior of the $\mathrm{H} \alpha$ line is similar to that observed in November 1996. An additional small peak in the center of a blue-shifted P Cyg absorption is also seen. It was situated at $-200 \mathrm{~km} \mathrm{~s}^{-1}$ during the entire season. The He I line displayed the inverse PCyg-profile during the first observing night on March 28, which completely disappeared three days later (on March 31). The lines of the DNa I doublet had four local blue-shifted absorption components, which demonstrated quite different positional changes over three days: the first had shifted from -205 to $-235 \mathrm{~km} \mathrm{~s}^{-1}$, the second remained constant in position $\left(-150 \mathrm{~km} \mathrm{~s}^{-1}\right)$, the third moved from -110 to $-95 \mathrm{~km} \mathrm{~s}^{-1}$, and the fourth (the least of them) was also seen at constant velocity $-60 \mathrm{~km} \mathrm{~s}^{-1}$.

The widths of the lines changed slightly in comparison to the previous observing season: the $\mathrm{H} \alpha$, He I and DNa I profiles ranged respectively between $\pm 450, \pm 370$, and from -300 to $+250 \mathrm{~km} \mathrm{~s}^{-1}$.

\subsubsection{March-April 1998}

This observing season was marked by a drastic strengthening of the secondary blue-shifted emission on the PCyg-profiles of $\mathrm{H} \alpha$ and DNaI, which started on March 30 (Figs. 1e-h). 


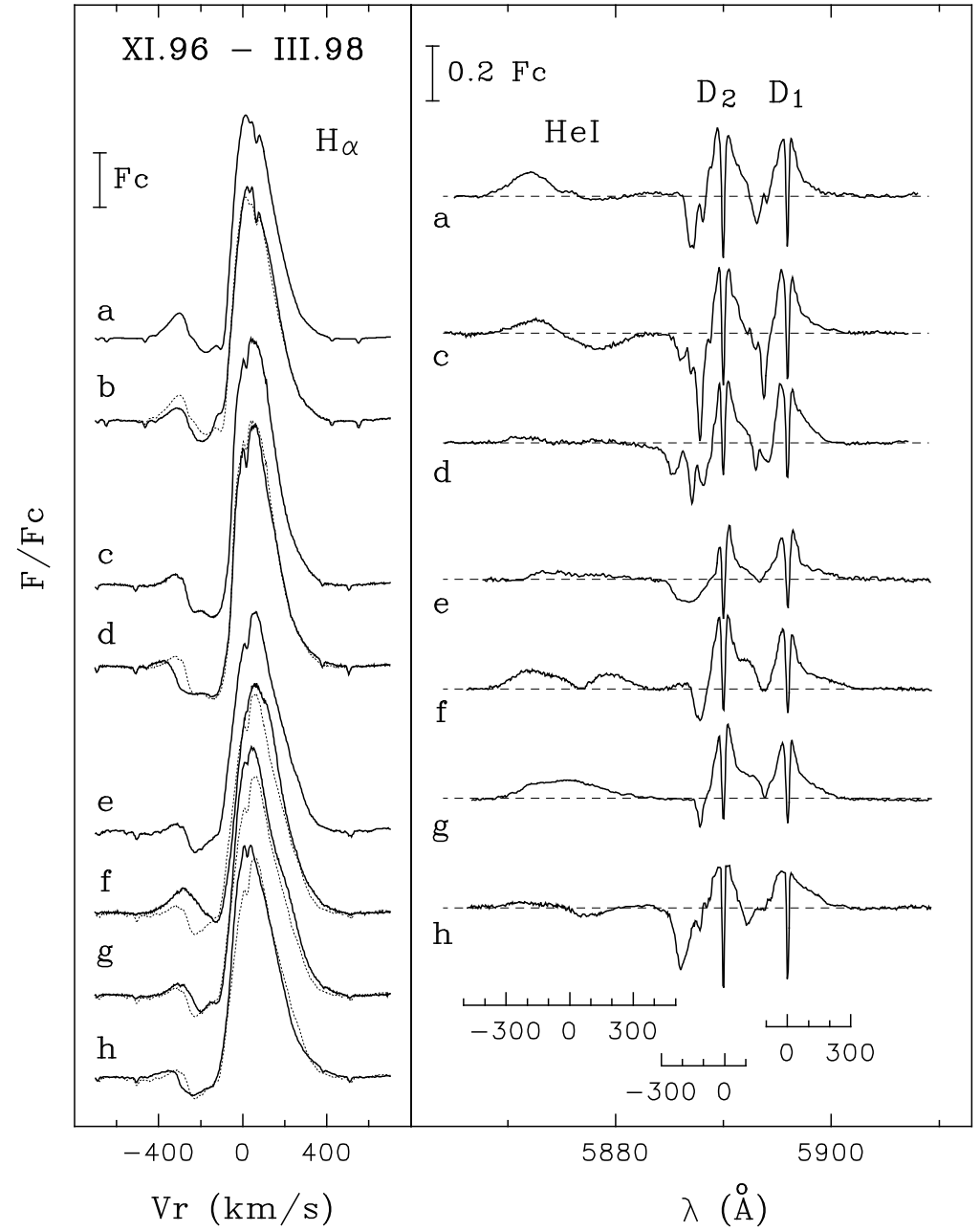

Fig. 1. Nightly mean $\mathrm{H} \alpha$, He I, and DNa I profiles observed in HD 31648 on the following dates: a) $1 / 2.11 .96$; b) $5 / 6.11 .96$; c) $28 / 29.03 .97$ d) $31.03 / 1.04 .97$; e) $28 / 29.03 .98$; f) $30 / 31.03 .98$; g) $31.03 / 1.04 .98$; h) $3 / 4.04 .98$. The radial velocity scale is given with respect to the star. For the sake of comparison, the $\mathrm{H} \alpha$ profiles obtained on 1/2.11.96, 28/29.03.97, and 28/29.03.98 are also superimposed as dotted lines on the profiles of the consequent observing nights. The dashed lines in the right panel show the continuum level.
This new spectral feature was seen in $\mathrm{H} \alpha$ during only one night and at least two nights in DNa I (March 30-31).

Simultaneously with this transformation of the $\mathrm{H} \alpha$ and DNa I profiles, striking variability was observed in the He I line. On March 28 this line was almost not seen at all. Two isolated emission peaks appeared on March 30 separately at negative and positive velocities. On March 31 the peaks merged into a single emission profile, which practically disappeared again three days later, with a weak red absorption still seen (April 3).

A system of local spectral features was formed in the blue part of the $\mathrm{H} \alpha$ and DNaI profiles near the end of the phase of their transformation. It is remarkable that the features at -200 and $-115 \mathrm{~km} \mathrm{~s}^{-1}$ were observed both in $\mathrm{H} \alpha$ and DNa I with their positions remaining constant over four days (from March 30 to April 3).

The profile widths of the $\mathrm{H} \alpha$ and DNa I lines (including the newly formed blue emission) and of the He I line (only during the active phase on March 30-31) were approximately the same as a year ago.

\subsubsection{October 1998}

The most large-scale variations of the $\mathrm{H} \alpha$ profile were detected in October 1998. Looking like a P Cyg III-type profile on October 20, the profile started its global transformation towards the PCyg II type on the next night (October 21). During subsequent observing dates (October 23-25), the profile became a typical P Cyg II shape with its central emission peak being $30 \%$ more intense than that seen on October 20. A system of local spectral structures superimposed on the main blue-shifted absorption component developed in the profile (Fig. 2). A similar behavior was shown by the $\mathrm{H} \beta$ line, but without significant intensity changes (Fig. 4).

At the same time, the He I line profile displayed a distinctive structure with a rather stable emission peak at negative velocities and a variable red absorption which transformed to a single emission peak on October 21. During this season the blue wing of the line profile was much more extended in comparison to the red one (up to -400 against $+200 \mathrm{~km} \mathrm{~s}^{-1}$ ).

The DNa I lines demonstrated very complex behavior. A number of strongly variable absorption features were observed in the blue part of the profile. Some of them were rather deep and narrow, others looked like wide depressions extended towards the blue to $-500 \mathrm{~km} \mathrm{~s}^{-1}$. During this season the red edge of the DNa I lines did not exceed $+250 \mathrm{~km} \mathrm{~s}^{-1}$.

\subsubsection{March 1999}

This observing season is characterized by an unusual type of He I line profile looking like a single emission peak with 

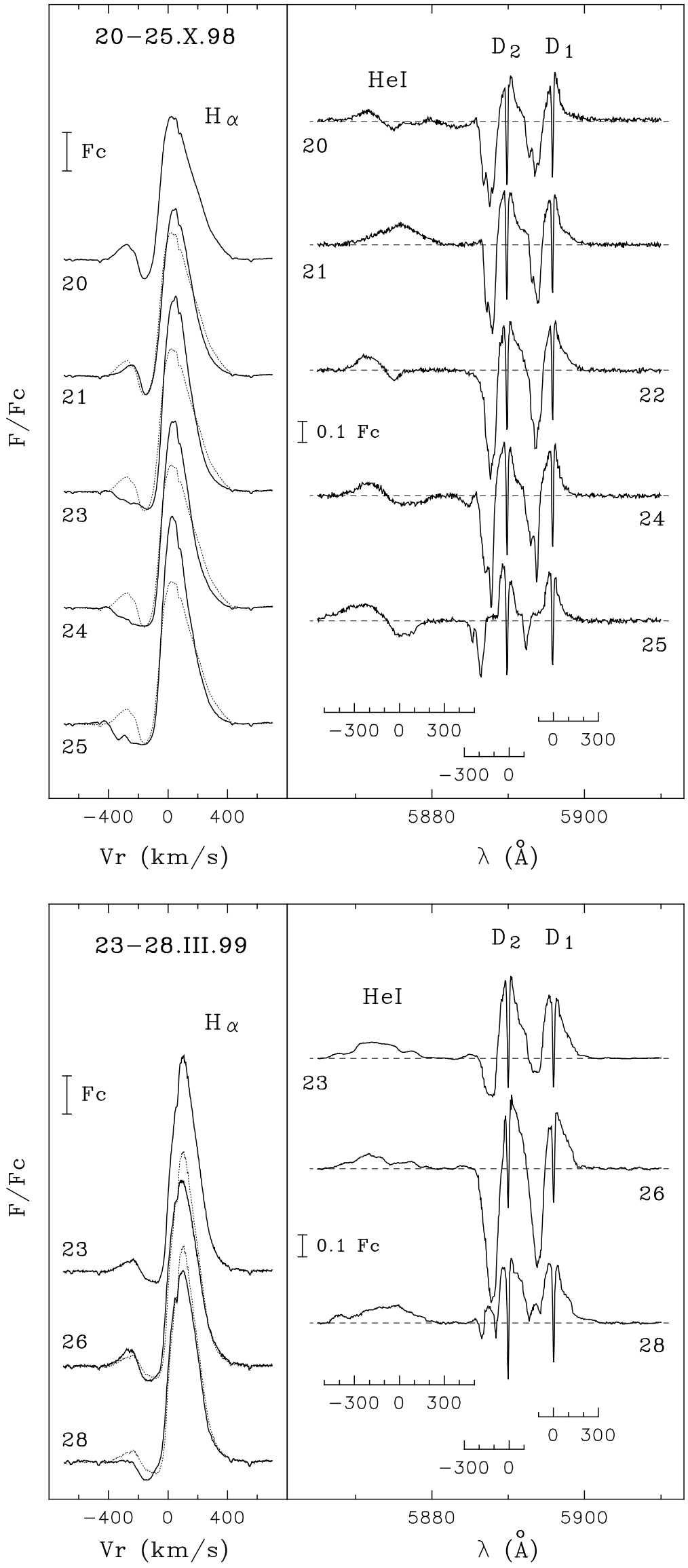

Fig. 2. The same as Fig. 1, but for October 1998. Dates of observations are indicated in the figure. The dotted line shows the $\mathrm{H} \alpha$ profile obtained on 20.10 .
Fig. 3. The same as Fig. 1, but for dates: a) 23/24.03.99; b) $26 / 27.03 .99$; c) $28 / 29.03 .99$. The dotted line shows the $\mathrm{H} \alpha$ profile obtained on 23.03 . 


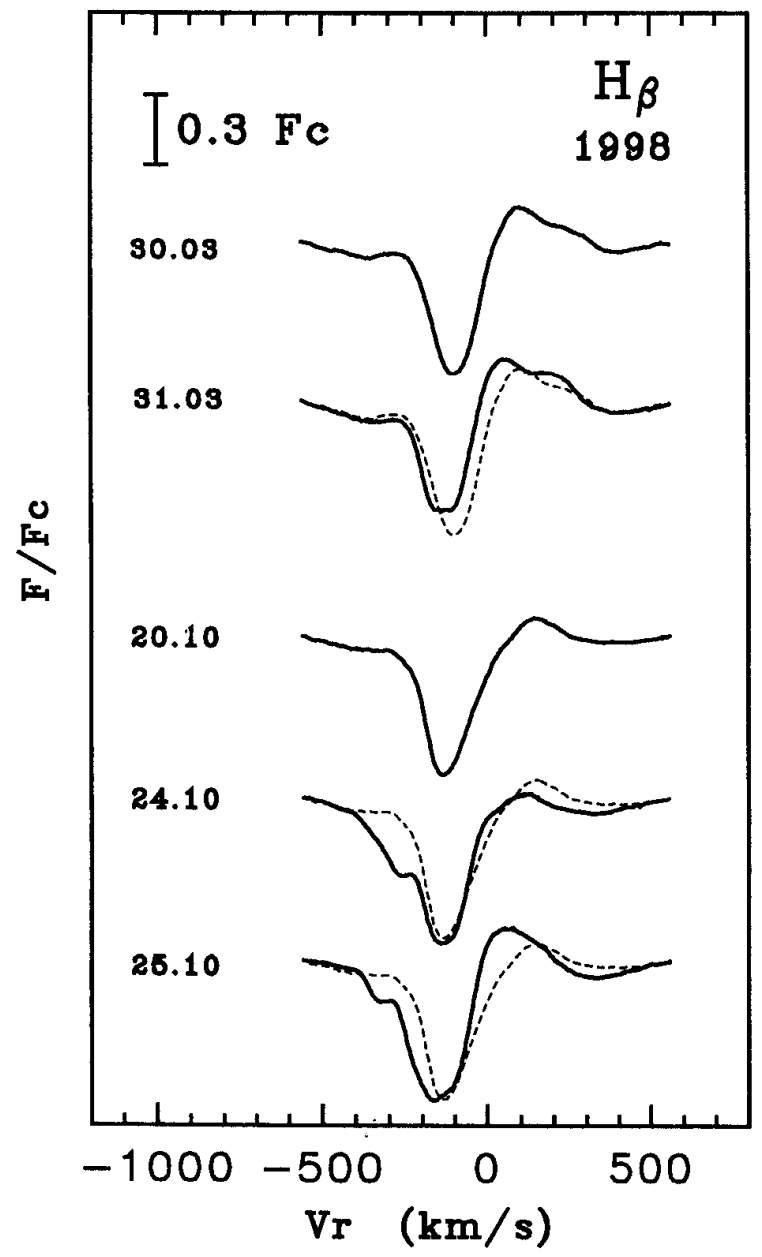

Fig. 4. Nightly mean $\mathrm{H} \beta$ profiles observed in HD 31648 in 1998. Dates of observations are indicated in the figure. The dashed lines show the profiles obtained on 30.03 and 20.10 which are superimposed on the profiles of the respectively next observing nights.

uneven outlines and the blue wing being anomalously extended (up to $-500 \mathrm{~km} \mathrm{~s}^{-1}$ ) whereas the red edge of the profile remained not further than $+200 \mathrm{~km} \mathrm{~s}^{-1}$ (Fig. 3).

The H $\alpha$ line shows a typical PCyg III-profile, but with a very intense central emission peak, similar to that observed in October 1998 after the global profile transformation.

The most variable regions in this season were the DNa I lines. On March 26 the blue-shifted P Gyg absorption became a factor of four deeper in comparison to that observed on March 23. Two nights later (on March 28), a strong additional emission bump overlapped almost the whole absorption component.

\subsection{Polarization features}

An analysis of the results of our polarimetric observations has been performed in terms of the normalized Stokes parameters $q$ and $u$. These parameters fit the normal distribution that guarantees the correctness of procedures of their averaging (see, e.g., Shakhovskoy 1994, and references therein). Besides that, the analysis of the polarization parameters on the $(q, u)$-plane is the

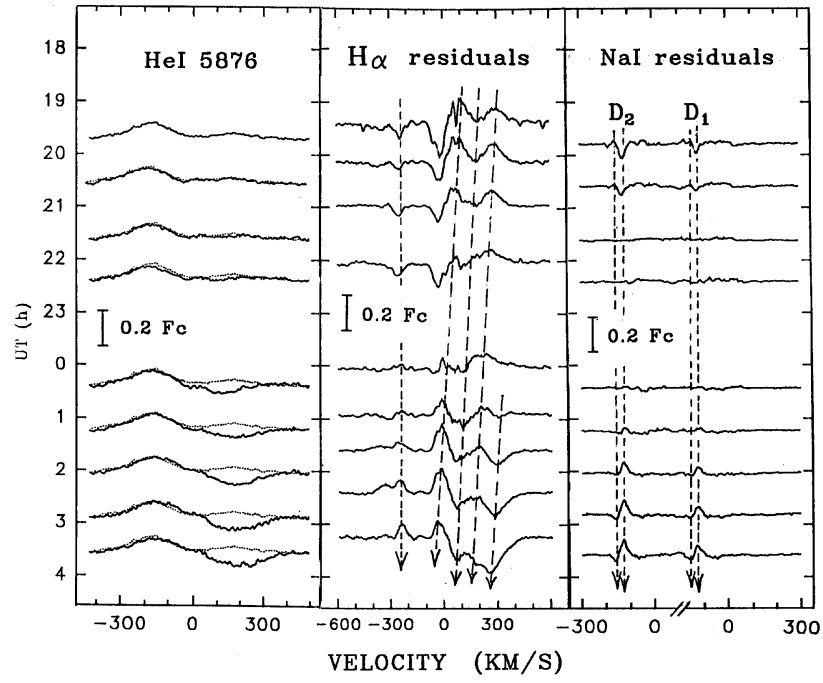

Fig. 5. Rapid variations of the $\mathrm{He}$ I, $\mathrm{H} \alpha$, and $\mathrm{DNa}$ I line profiles observed in HD 31648 during the night 1/2.11.96. The dashed lines show the first in the series He I line profile superimposed on the other profiles for comparison (left). The residuals with respect to the nightly mean profile are given for the $\mathrm{H} \alpha$ and DNa I lines (middle and right). The short-dashed and long-dashed lines indicate velocity positions for respectively "standing" and "moving" waves on the profiles.

most illustrative way to separate different sources of polarized radiation and to compare their properties.

The observed Stokes parameters of HD 31648 demonstrate significant variations from date to date. The character of this variability being rather irregular, we started with analysis of the non-variable polarization components. On the $(q, u)$-plane the points corresponding to the weighted mean values for all the data in each passband are situated along a straight line (Fig. 6). This allows us to present each point as a sum of two constant components, determined by means of the least-squares method (Fig. 6, top): a grey component with $P=0.56 \%$ and $\theta=61.5 \pm 1.0$, and a selective one with $P(\lambda) \propto \lambda^{-1}$ and $\theta=148^{\circ} .8 \pm 1.2$, shown in Fig. 6 (bottom). A similar two-component structure of the averaged linear polarization was observed in another object of our programme, the Herbig Ae star HD 36112, located in the same complex of dark clouds as HD 31648 (Beskrovnaya et al. 1999).

Since the IS polarization has a different wavelength dependence, one can conclude that the contribution of the IS component to the observed polarization parameters of HD 31648 is rather small. This is to be expected, taking into account the small value of reddening towards the object (see Sect. 1.2).

A detailed analysis of polarimetric variability of HD 31648 is rather complicated because of small values of the polarization parameters and uneven data sampling. The most precise data were obtained during the only long continuous series of observations in November 1996. The variability of the Stokes parameters observed during this season is illustrated in Fig. 7. In all the passbands (except for $U$ ) the amplitude of variations is sufficiently larger than the errors at the $3 \sigma$-level (the error bars indicate $\pm \sigma$-level). The variations of both $q$ and $u$ are rather similar in different passbands and are characterized by long-term trends shown in the figure by solid lines. A notable 


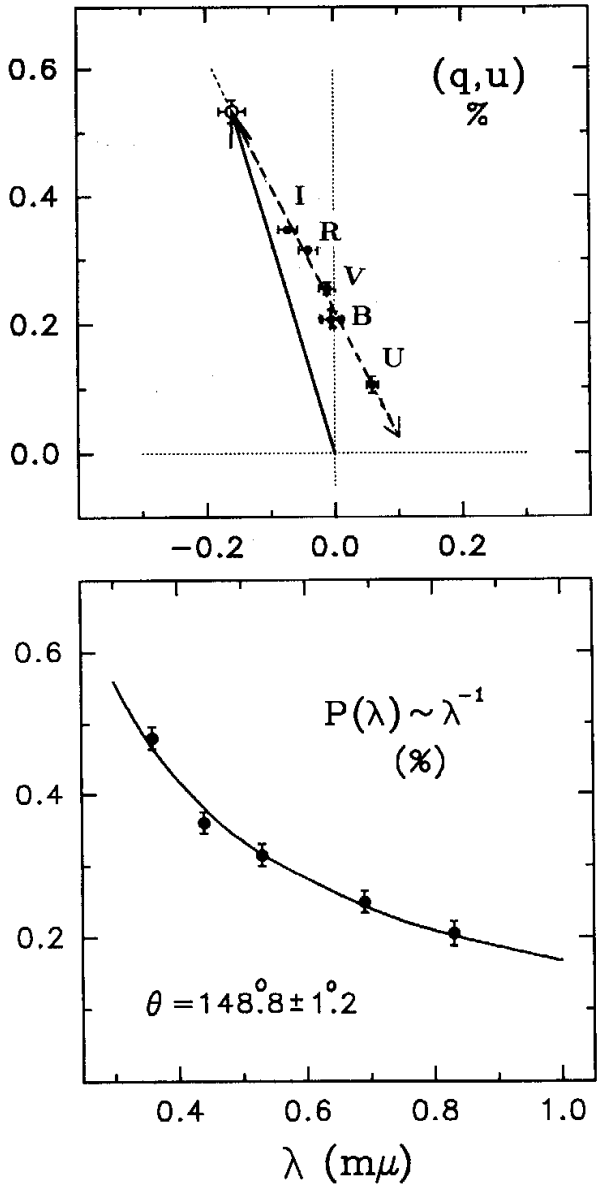

Fig. 6. Top: the Stokes parameters of HD 31648 in different passbands averaged for all observing seasons (filled circles). The open circle represents the end of the grey component vector (with error bars from the least squares method) and the initial point of the selective component vectors. The solid and dashed arrows indicate the vector of the grey component and the direction of the selective component (the same in all the passbands), respectively. The error bars in both panels show $\pm \sigma$-level. Bottom: the wavelength dependence of the selective component. The line is drawn for the law $P(\lambda) \propto \lambda^{-1}$.

break of the trends near November, 6 is clearly seen in the $V$, $R$, and $I$ passbands.

The traces of these trends on the $q, u$-plane have a form of an arc (Fig. 8). The direction of moving along the trace is the same for all the passbands, the shape of arcs is also the same except for $U$, where the accuracy is worse. For the $V, R$, and $I$ passbands the traces contain a jump, dividing the arc into two different fragments.

The similar arc-like traces of the polarization parameters in the $q, u$-plane have been also revealed in AB Aur (Beskrovnaya et al. 1995) and HD 36112 (Beskrovnaya et al. 1999).

\section{Discussion}

\subsection{Large-scale structure of the envelope}

According to common views, the circumstellar envelopes of the Ae Herbig stars, similar to HD 31648, contain the equatorial gaseous-dusty disk and the stellar wind at higher latitudes.

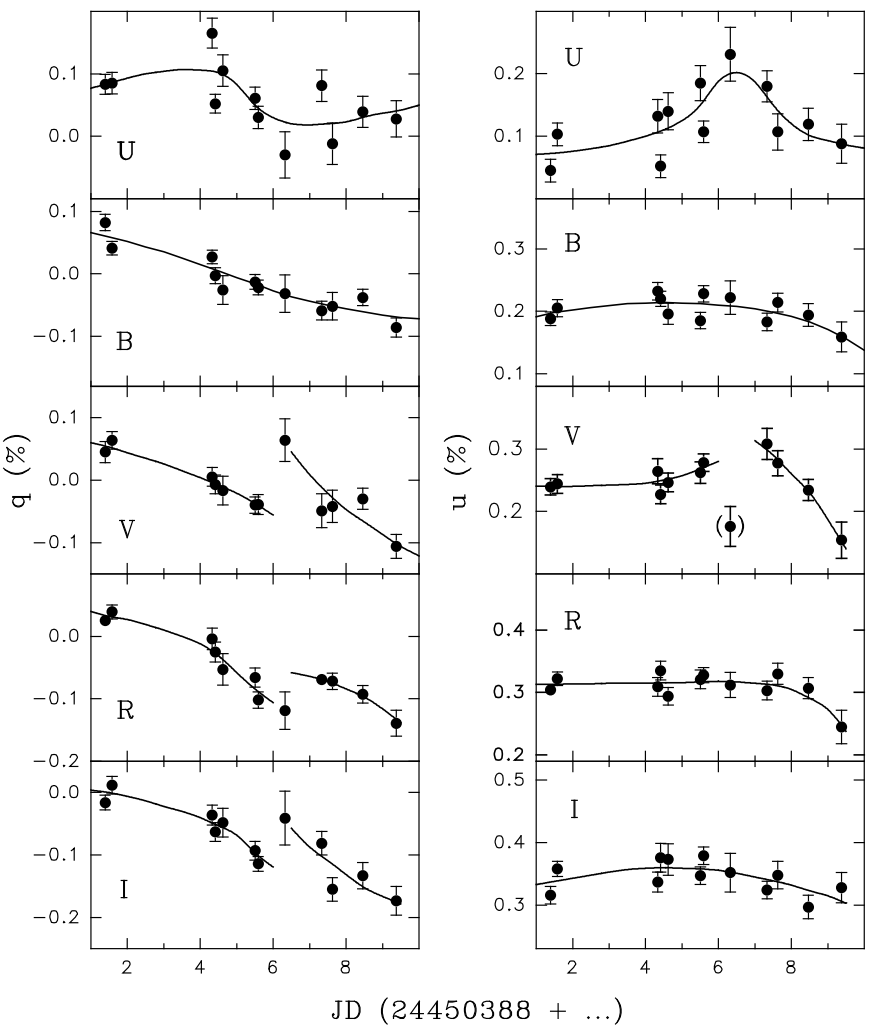

Fig. 7. Variations of the polarization $q, u$-parameters of HD 31648 detected in the UBVRI-passbands in November 1996. The solid lines show trends of variations driven by means of the median-method.

The large-scale manifestations of these main circumstellar components can be directly observed in the radio, IR, Ly $\alpha$, etc., by means of imaging techniques and have a form of elongated features with perpendicular bipolar outflows.

Observations of HD 31648, described in our paper, allow us to study properties of the accretion disk and the stellar wind, as well as an interconnection between these two features in the vicinity of the star. A search for temporal correlations between observational manifestations of the inflow and outflow phenomena is especially important because it helps to clarify the question about the nature of wind generation in the HAEBEs, which remains a matter of debate to date. Information on the peculiarities of the accretion process can be obtained from the analysis of the red part of the He I $\lambda 5876 \AA$, while the properties of the stellar wind can be revealed from the behavior of the blue part of this line and, mainly, from the emission $\mathrm{H} \alpha$ and $\mathrm{H} \beta$ profiles. Additional information on the dust properties is provided by the polarimetric and photometric data.

\subsubsection{Observational manifestations of the CS disk}

As mentioned in Sect. 1.2, the CS disk has been directly detected in the radio maps of the HD 31648 region (Mannings et al. 1997). Evidence for a rotating scattering disk-like feature around HD 31648 has been also presented by Vink et al. (2002) on the basis of their spectropolarimetric scanning across the $\mathrm{H} \alpha$ profile. 
The data presented in our paper confirm the existence of the disk and provide some additional information about its properties.

1. A noticeable asymmetry of the DNa I line profiles in the spectrum of HD 31648 with a dominating blue component was observed during some observing seasons (in particular, in October, 1998). This effect can be caused by the existence of optically thick matter in the remote part of the envelope, which is concentrated towards the equatorial plane and screens the receding part of the stellar wind. The same phenomenon has been also found in HD 50138 (Pogodin 1997) and HD 36112 (Beskrovnaya et al. 1999).

2. According to the results of our polarimetry (see Sect.3.3), the vectors corresponding to the selective and non-selective components (Fig. 6, top) have practically the opposite directions in the $(q, u)$-plane. In projection onto the sky plane, the position angles (PA) of these two vectors are $149^{\circ}$ and $53^{\circ}$, respectively. Taking into account that the dust region on the radio-map of HD 31648 is elongated along $\mathrm{PA}=157_{-3}^{+4}$ degrees (Mannings et al. 1997), one can conclude that the selective component is polarized in the disk plane, while the grey one - in the perpendicular plane. The two-component structure of the intrinsic linear polarization of HD 31648 requires additional investigation on the properties of scattering media surrounding this object. In the first approximation, it can be explained suggesting that the disk contains both large grains (responsible for the grey component) and small elongated particles (responsible for anomalous selective component, polarized in the disk plane) (for details, see Dolginov et al. 1995).

3. The red part of the HeI $\lambda 5876 \AA$ line, which is an indicator of the accretion process from the CS disk onto the star, is strongly variable in HD 31648. It has been observed in both absorption and emission (see Sect. 3.2) and its behavior resembles previous objects of our program (HD 163296, HD 50138, HD 100546). Variations in the red part of the HeI $\lambda 5876 \AA$ line in the spectra of Herbig Ae stars are likely to be related to a discrete character of accretion onto these objects (Grady et al. 1996), that results in irregular fluctuations of physical conditions in the high-temperature zone, where this line is forming. In contrast to other HAEBEs of similar type, the blue part of the He I $\lambda 5876 \AA$ profile in the spectrum of HD 31648 forming near the base of the stellar wind is also very variable.

A possible relationship between processes of accretion and outflow in the envelope of the object is addressed in Sect. 4.1.3.

\subsubsection{Latitudinally inhomogeneous stellar wind}

The most pronounced spectral indicator of the stellar wind is the first members of the Balmer series $\mathrm{H} \alpha$ and $\mathrm{H} \beta$. The intensities, as well as a character of variations of the emission $\mathrm{H} \alpha$ and $\mathrm{H} \beta$ lines in the spectrum of HD 31648 resemble those observed in another Ae Herbig star, HD 163296 (Pogodin 1994; Beskrovnaya et al. 1998, and references therein). The profiles of these lines are predominantly of the P Cyg III type, which can be occasionally transformed into the P Cyg II (see Sect. 3.2.4).

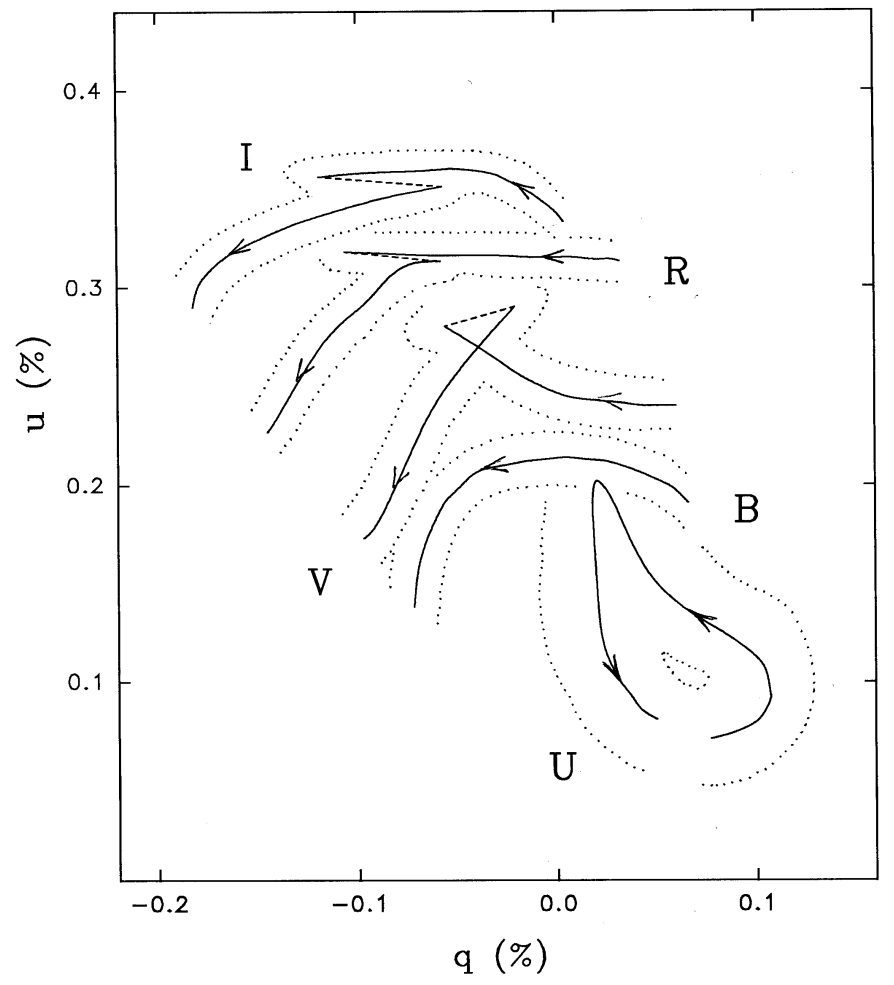

Fig. 8. Trends of the polarimetric variability of HD 31648 in the UBVRI - passbands on the $q, u$-plane which were observed in November, 1996. Arrows indicate the direction of time increasing. The dotted lines mark the error levels $( \pm \sigma)$ for each trend, which were determined by means of the least-squares method.

According to radio-mapping data, these two stars seem to be seen at different inclination angles $i: 58^{\circ}$ for HD 163296 (Mannings \& Sargent 1997) and about $30^{\circ}$ for HD 31648 (Mannings et al. 1997). In spite of these differences, the spectroscopic properties of these two objects are very similar. This means that they are determined by a factor common for these stars, namely, that the inclination of their disk plane to the lineof-sight is intermediate between pole-on and edge-on. This assertion is also confirmed by the low photometric activity of the stars (see Sect. 3.1).

For objects with such an orientation, the line-of-sight intersects the wind zone at middle latitudes. If the outflowing gas is dense enough, an absorption component is seen in the blue part of the emission line profile forming a typical PCyg-type structure. The equatorial disk and the wind portion which is not intersected by the line-of-sight contribute to the emission peak. We assume that a redistribution of matter inside the wind zone can be responsible for global transformations of the PCygtype $\mathrm{H} \alpha$ profile observed in Herbig Ae stars like HD 163296 and HD 31648.

In general, the problem of structural peculiarities of the wind is closely connected with the question about possible configuration of the stellar and disk magnetic fields powering the motion and distribution of SC gas. Currently, two main groups of physical (MHD) models can be distinguished with regard to the spatial structure of the PMS stellar envelopes. 


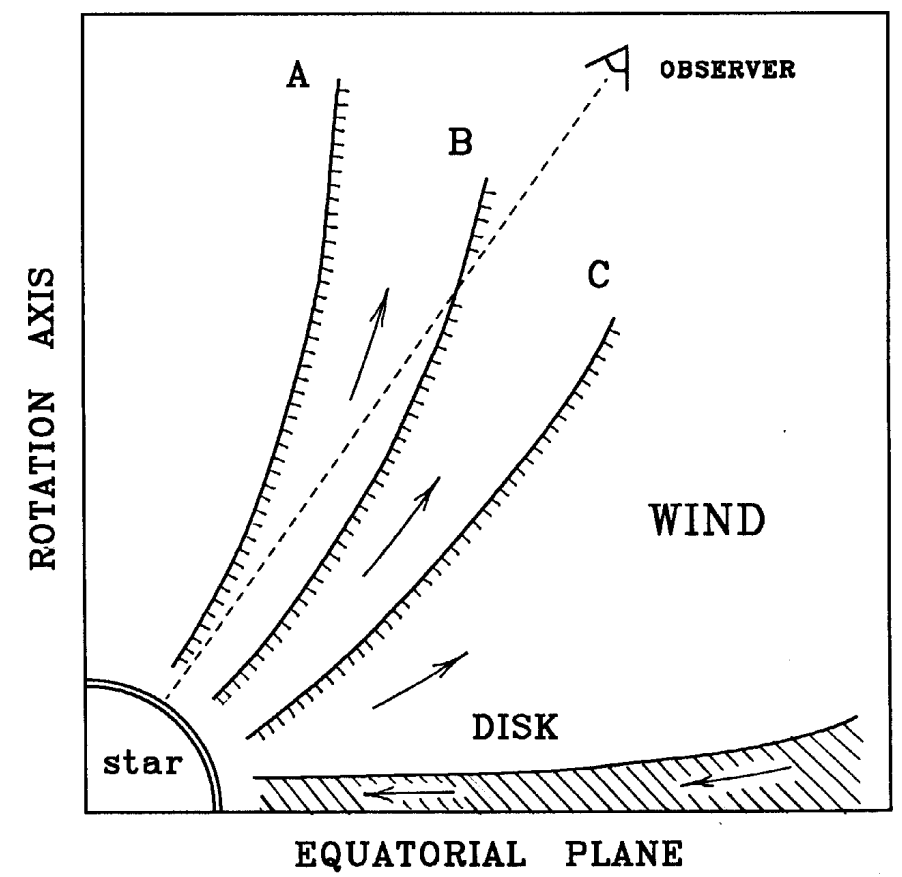

Fig. 9. Schematic geometry of the envelope near a Herbig Ae star in the form of a meridional section through the rotation axis. The equatorial accretion disk and the high-latitude boundary of the wind zone in three positions A, B and C are displayed. The cases A, B and C correspond, respectively, to three types of the line profile, usually observed in HAEBEs: A-P Cyg II; B-P Cyg III; C-a single peak. The arrows indicate directions of gaseous flows in the disk and the wind.

The first group combines the following models: a) an accretion-magnetospheric approach (Camenzind 1990; Hartmann et al. 1994; Shu et al. 1994), b) models of the wind flowing directly from the disk (e.g. Königl 1996), and c) models of the wind generating from the stellar surface independently of the accretion process (Strafella et al. 1998). In spite of the opposite concepts of the wind origin, all these models predict that at small distances from the star the region of stellar wind is situated at lower latitudes at both sides of the equatorial accretion disk. The stellar wind flows close to the disk in the vicinity of the star and turns along the polar axis only at larger distances (tens of $R_{\star}$ ), where a polar collimation of the wind occurs due to azimuthal (pinch) stresses and the polar jets are formed. The driving mechanism of the wind near the star is connected to the centrifugal force accelerating the CS gas along the open magnetic force lines while they are rotating.

The second group of models is considered in a number of theoretical articles (e.g. Turner et al. 1999; Goodson et al. 1999) devoted to the formation of polar jets from young stellar objects near the star in addition to the main wind at middle latitudes. These MHD-calculations have been performed only for low-mass protostars and T Tau stars with strong magnetic fields. The applicability of this approach to the HAEBEs is not clear to date.

We attempt to explain the large-scale variability of the $\mathrm{H} \alpha$ and $\mathrm{H} \beta$ profiles in the spectrum of HD 31648 in the framework of a simplified geometric model based on the physical concepts of the first group, assuming the bulk of the wind near the star to flow at middle latitudes. Otherwise (in the case of polar collimation of the wind), the observed properties of such differently oriented objects as HD 31648 and HD 163296 would show many more dissimilarities. The sketch of the model is given in Fig. 9. The wind zone is placed at low and middle latitudes outside the equatorial accretion disk. If the opening angle is large enough for the line-of-sight to intersect the wind, a P Cyg profile is formed (cases A, B). In the opposite case, no absorption component can be produced, and the line appears as a single emission peak (case C).

The P Cyg profile of type II is observed if the stellar limb is completely screened from an observer by the wind region (case A). If the limb is screened only partly, the PCyg III-type profile will be seen (case B). This is connected to a specific velocity law which is established in the wind as a result of the magnetic centrifugal action. This mechanism accelerates the CS gas up to hundreds $\mathrm{km} \mathrm{s}^{-1}$ within a restricted region near the star; further away the wind is decelerating with distance. This effect is clearly seen if we compare the width of the remote CS DNa I lines typical for the spectra of Herbig Ae stars with that of the emission $\mathrm{H} \alpha$ line originating throughout the envelope including a region where the wind velocity reaches its maximum. As a rule, the latter can be as large as $\pm 400-500 \mathrm{~km} \mathrm{~s}^{-1}$, while the width of the DNa I lines does not exceed $\pm 200 \mathrm{~km} \mathrm{~s}^{-1}$. In the case B (see Fig. 9), the stellar limb is hidden from an observer only by an external part of the wind, where the outflow velocity is rather low. Thus, the blue absorption component of the observed P Cyg line profile should be narrower than in the case A (P Cyg II-type), since it originates beyond the region of large velocities.

As applied to HD 31648, this picture is justified by the fact that the observed $\mathrm{H} \alpha$ profiles in the spectrum of this object have, as a rule, the P Cyg III type, whereas the DNa I lines originating only in the outer wind show the P Cyg II profiles.

This approach allows one to explain qualitatively the observed profile variations. A quantitative modelling and accurate determination of structural and kinematical parameters of the envelope is beyond the scope of this work. This task is multiparameter and cannot be solved unambiguously. Besides that, these parameters strongly depend on a concrete physical mechanism of wind generation which still remains to be understood.

Nevertheless, some conclusions on the latitudinal distribution of different wind portions can be made on the example of the most prominent event in October, 1998 (Sect. 3.2.4). In the frame of our concept, the observed profile variation may be connected to additional generation of stellar wind at higher latitudes, resulting in an increase of the emitting gas mass. It is noteworthy, that while during this period the $\mathrm{H} \alpha$ emission line has got more intense, the $\mathrm{H} \beta$ line remains practically constant (Fig. 4). Therefore, the appearance of additional high-latitude wind was followed by an increase of the observed Balmer decrement. According to the results of previous modelling of CS gaseous media (Pogodin 1986), this effect can be caused by a decrease of the average gas density in the wind after its expansion towards higher latitudes. Thus, the hypothetical new high-latitude wind has to be of significantly lower density than that at low and middle latitudes. The main 
contribution of the additional wind to the emission part of the profile is limited to $\pm 200 \mathrm{~km} \mathrm{~s}^{-1}$, while the total width of the main P Cyg profile is about $\pm 400 \mathrm{~km} \mathrm{~s}^{-1}$ (Fig. 2). This fact is in favor of the assumption that it was generated at higher latitudes where the accelerating magnetic centrifuge is less effective.

We assume that the latitudinal redistribution of the outflowing matter might result from structural changes of the magnetic field configuration in the region of star-disk interaction.

Another approach to the interpretation of the large-scale $\mathrm{H} \alpha$ and $\mathrm{H} \beta$ profile variability in the Herbig Ae stars assigns these variations to the jet activity of the star. This mechanism can be considered if the jets are clearly detected not only in the resonance and forbidden lines, but also in the subordinate line $\mathrm{H} \alpha$ (this will prove that the dense polar jets can form just in the vicinity of the star). In our phenomenological model the jets are features of rather low density. They can be visible in the $\mathrm{H} \alpha$ line, but cannot stimulate its large-scale variability. They can also be seen in the resonance DNa I doublet and the striking variability of its components (see Sect. 3.2) might be connected, to a great extent, to the jet activity of HD 31648 .

However, the question remains to be clarified concerning the factors which could be responsible for hypothetical structural changes in both the stellar wind and the configuration of global magnetic field as well as for the jet activity of the object. Among possible causes the changes in the disk accretion regime can be assumed. Information about disk-to-wind relations can be obtained from the analysis of correlation between variations in the "wind line" $\mathrm{H} \alpha$ and the "accretion line" He I $\lambda 5876 \AA$.

\subsubsection{Correlations between the disk and wind manifestations}

A search for an expected correlation between variations in the $\mathrm{H} \alpha$ and $\mathrm{He}$ I lines, observed from HD 31648, allows us to distinguish three different time periods.

The first of them covers three observing seasons from November, 1996 to April, 1998 (see Fig. 1). During this period the $\mathrm{H} \alpha$ line shows only small variations near the central emission peak and in the region of PCyg III absorption/emission feature. At the same time, the He I line is strongly variable both in the red and blue parts. A correlation between changes in the $\mathrm{H} \alpha$ and the He I lines can barely be found.

During the second period (October 1998), a remarkable global transformation of the $\mathrm{H} \alpha$ line profile was observed on October 21-23 (see Sect. 3.2.4). This event can be interpreted within the model of latitudinal expansion of the wind zone. At the beginning of this transformation, an emission suddenly developed in the red part of the He I line profile, just where an absorption was seen a day before and would be observed two days later. This phenomenon is likely to be related to an episode of fast enhancement of accretion from the disk onto the star. A relation of this discrete infall event to the structural changes in the wind can be suspected. During this episode, the accretion event occurs before the transformation of the wind structure. Therefore, we can suggest that at least during this observing season changes in the accretion regime have influenced the wind properties.

In the third period (March 1999), neither $\mathrm{H} \alpha$ nor He I lines showed significant profile variability (Fig. 3). The helium line was observed exclusively in emission throughout the season. It is remarkable that the central emission peak of the $\mathrm{H} \alpha$ line is usually more intense provided the red part of the He I line is observed (or was observed just before) in emission (October 22-25, 1998; March 1999).

\subsection{CS inhomogeneities}

Information on the presence and properties of local inhomogeneities forming in different parts of the CS envelope of HD 31648 has been provided by the study of: a) polarimetric variability; b) rapid variability of the $\mathrm{H} \alpha$ line profile; and c) behavior of local spectral features in the blue part of the DNa I and $\mathrm{H} \alpha$ lines.

\subsubsection{Dusty condensations}

Investigation of the polarization parameters has revealed the presence of dusty condensations rotating in the CS envelope. As seen in Fig. 8, the variations of the Stokes parameters during 7 observing nights in November, 1996 in each passband correspond to the counter-clockwise motion along arclike tracks on the $(q, u)$-plane. These tracks are similar in shape, size, and orientation for four passbands, where the polarimetric accuracy is higher ( $B, V, R, I$-bands). The direction of motion along the track is approximately orthogonal to the vectors of the constant polarization components. This pattern allows us to conclude that polarimetric variability of HD 31648 during this season was caused by the rotation of a local dusty condensation, yielding non-selective polarization effect. The obvious interruption of the tracks, which is seen in the $V, R, I$-passbands, shows that there could be more than one condensation.

\subsubsection{Moving inhomogeneities in the wind}

In Sect. 3.2.1 we discuss different types of rapid variability observed in the $\mathrm{He} \mathrm{I}, \mathrm{H} \alpha$, and DNa I line profiles on November 1 , 1996. A complex pattern of $\mathrm{H} \alpha$ variations (Fig. 5, middle) is almost identical to that observed in another Herbig Ae star, HD 163296 (Fig. 5b in Pogodin 1994). Positionally stable but variable in intensity local spectral features are present at negative velocities ("standing waves"), whereas "moving waves" are clearly seen in both red and central parts of the profile. Similar features in form of "standing waves" have been also observed in the blue part of the DNa I profiles (Fig. 5, right). Taking into account all the arguments presented for an explanation of the rapid spectral variability of HD 163296 (Pogodin 1994; Beskrovnaya et al. 1998), we conclude that, by analogy with HD 163296, the envelope of HD 31648 is likely to contain stream-like azimuthal inhomogeneities in the stellar wind, responsible for an appearance of positionally stable spectral bumps in the blue part of the $\mathrm{H} \alpha$ and DNa I profiles, as well as local density condensations ("blobs") in the wind 
acceleration zone, which manifest themselves in the red part of the $\mathrm{H} \alpha$ profile.

\section{Conclusions}

The results of our complex programme of spectral, photometric, and polarimetric study of the Ae star HD 31648 have shown that this star displays many structural and kinematical peculiarities of its CS envelope common with other young HAEBEs. The most important of them are as follows:

1. Large-scale variability of the Balmer line profiles from P Cyg IIIto P Cyg IIas an indicator of changes in the latitudinal distribution of stellar wind.

2. The presence of a high-temperature zone responsible for the observed He I line, providing information on accretion phenomena.

3. Short-term variability of the $\mathrm{H} \alpha$ and $\mathrm{DNa}$ I line profiles probably connected to formation of CS inhomogeneities like outflowing jets, dense gaseous condensations, and latitudinal layers in the stellar wind zone.

4. Variable multi-component polarization originating in the CS environment due to a) the presence of dust particles of different size and form; and b) motion of local dusty condensations.

Some dissimilarities have been revealed in the behavior of the HeI line in the spectrum of HD 31648 and other Herbig Ae stars. The blue emission peak on the profile of this line is more variable. This fact is possibly connected to the orientation of the star+envelope system relative to the line-of-sight at a rather small inclination angle.

A relation between variability of the $\mathrm{H} \alpha$ and $\mathrm{He}$ I lines in October, 1998 has been suspected. This favors the assumption that changes in the accretion regime can affect the spatial structure of the wind zone. A detailed study of this relation can provide important information on the origin and characteristics of the star-disk-wind-interaction in HAEBEs.

Acknowledgements. We would like to thank Yu.N.Gnedin, Yu.S.Efimov, N.A.Silant'ev and R.V. Yudin for useful comments concerning the polarimetric part of this study. A.S. Miroshnichenko has placed at our disposal the additional photometric data obtained in 1995-1996. This work was sponsored by the Program "Integration" (grant KO232), State Scientific and Technical Program "Astronomy" and Program of the Presidium of the RAS "Non-stable phenomena in astronomy". N. Beskrovnaya acknowledges the support of the Russian Foundation for Basic Research under Grant N 03-02-17223a.

\section{References}

Allen, D. A., \& Swings, J. P. 1976, A\&A, 47, 293

van den Ancker, M., de Winter, D., \& Tjin A Djie, H. R. E. 1998, A\&A, 330, 145
Beals, C. S. 1951, Pub. Dom. Astr. Obs., 9, 1

Beskrovnaya, N. G., Pogodin, M. A., Shcherbakov, A. G., \& Tarasov, A. E. 1994, A\&A, 287, 564

Beskrovnaya, N. G., Pogodin, M. A., Najdenov, I. D., \& Romanyuk, I. I. 1995, A\&A, 298, 585

Beskrovnaya, N. G., Pogodin, M. A., Yudin, R. V., et al. 1998, A\&AS, 127,243

Beskrovnaya, N. G., Pogodin, M. A., Miroshnichenko, A. S., et al. 1999, A\&A, 343, 163

Burbidge, M., \& Burbidge, G. R. 1954, APJ, 119, 501

Camenzind, M. 1990, Rev. Mod. Astron., 3, 234

Chen, P. S., Wang, X. H., \& He, J. H. 2000, Ap\&SS, 271, 259

Dolginov, A. Z., Gnedin, Yu. N., \& Silant'iev, N. A. 1995, in Propogation and polarization of radiation in cosmic media (Gordon and Breach Publ.), 352

Efimov, Yu. S., Piirola, V., \& Shakhovskoy, N. M. 1984, A\&A, 138, 62

Grady, C. A., Pérez, M., Talavera, A., et al. 1996, A\&AS, 120, 157

Grinin, V. P., Thé, P. S., Giampapa, M., et al. 1994, A\&A, 292, 165

Goodson, A. P., Böhm, K.-H., \& Winglee, R. M. 1999, ApJ, 524, 142

Hartmann, L., Hewett, R., \& Calvet, N. 1994, ApJ, 426, 669

Herbig, G. H. 1994, ASP Conf. Ser., 62, 3

Imhoff, C. L. 1994, ASP Conf. Ser., 62, 107

Jaschek, M., Jaschek, C., \& Andrillat, Y. 1988, A\&AS, 72, 505

Jaschek, M., Andrillat, Y., \& Jaschek, C. 1991, A\&A, 250, 127

Jaschek, M., Jaschek, C., \& Andrillat, Y. 1993, A\&AS, 97, 781

Königl, A. 1996, in Disks and outflows around young stars, ed. S. Beckwith, J. Staube, A. Quetz, \& A. Natta (Springer), 282

Kozlova, O. V., Grinin, V. P., \& Chountonov, G. A. 2003, Astrophysics, 46, 265

Mannings, V., \& Sargent, A. I. 1997, ApJ, 490, 792

Mannings, V., Koerner, D. W., \& Sargent, A. I. 1997, Nature, 388, 555

Merrill, P. V., Burwell, C. G., \& Miller, W. C. 1942, ApJ, 96, 15

Pérez, M. R., \& Grady, C. A. 1998, Space Sci. Rev., 82, 407

Piirola, V. 1975, Ann. Acad. Sci. Fennicae. Ser. A VI. Phys. N, 418, 61

Pogodin, M. A. 1986, Astrofizika, 24, 279

Pogodin, M. A. 1992, Sov. Astron. Lett., 18, 437

Pogodin, M. A. 1994, A\&A, 282, 141

Pogodin, M. A. 1997, A\&A, 317, 185

Pogodin, M. A. 2000, ASP Conf. Ser., 214, 344

Pogodin, M. A., Miroshnichenko, A. S., Bjorkmam, K. S., et al. 2000, A\&A, 359, 299

Reipurth, B., Pedrosa, A., \& Lago, M. 1996, A\&AS, 120, 229

Serkowski, K. 1974, N.Y. Acad. Press, 12, Pt.A., 301

Shakhovskoy, N. M. 1994, Izvestiya Crym. Astrophys. Obs., 91, 106

Shu, F., Naijta, J., Ostricer, E., et al. 1994, ApJ, 429, 781

Sitko, M. L., Savage, B. D., \& Meade, M. R. 1981, ApJ, 246, 161

Sitko, M. L. 1981, ApJ, 247, 1024

Sitko, M. L., Grady, C. A., \& Hanner, M. S. 1999, ApJ, 510, 408

Strafella, F., Pezutto, S., Corciulo, G. G., Bianchini, A., \& Vittone, A. A. 1998, ApJ, 505, 299

Thé, P. S., de Winter, D., \& Pérez, M. R. 1994, A\&AS, 104, 315

Turner, N. J., Bodenheimer, P., \& RóVzyczka, M. 1999, ApJ, 524, 129

Vieira, S. L. A., Pogodin, M. A., \& Franco, G. A. P. 1999, A\&A, 345, 559

Vink, J. S., Drew, J. E., Harries, T. J., et al. 2002, MNRAS, 337, 356 